\title{
A MACHINE LEARNING ENHANCED EMPIRICAL MODE DECOMPOSITION
}

\author{
D. Looney ${ }^{1}$ and D. P. Mandic ${ }^{1}$ \\ ${ }^{1}$ Imperial College London, UK \\ E-mail: \{david.looney06,d.mandic\}@imperial.ac.uk
}

\begin{abstract}
Empirical mode decomposition (EMD) is a fully data driven method for decomposing signals into a set of AM-FM components known as intrinsic mode functions (IMFs). Despite its usefulness in the analysis of real world signals, the process is rather deterministic and sensitive to parameters such as local envelope estimation. A combination of EMD and machine learning is proposed which provides an algorithm that is more robust to EMD parameters. In addition, the proposed extension is fully adaptive and facilitates the "data fusion via fission" mode of operation. The derivation and analysis of the proposed framework is supported with simulations in denoising and prediction applications.
\end{abstract}

Index Terms - empirical mode decomposition (EMD), machine learning, feature fusion, adaptive filtering

\section{INTRODUCTION}

The recently proposed EMD [1] is a fully data driven technique which decomposes the signal into narrow band components called intrinsic mode functions (IMFs). Unlike the Fourier based methods that project signals onto a fixed basis set, EMD is a unique signal decomposition tool that makes no prior assumptions about the data. It has proven to be particularly effective in the time frequency analysis of nonlinear and non-stationary data [1]. Recent work [2] illustrates that EMD provides a unifying framework for information fusion via fission (the phenomenon by which observed information is decomposed into a set of its components) for the purposes of signal restoration or alternatively to find relevant features within the data. However fusion via EMD has several limitations caused by potential errors in determining the IMFs and weak fusion procedures.

'Correct' estimation of the IMFs has long been a focus in the literature, since errors can arise from interpolation within envelope estimation, or an unsuitable choice of stopping criteria. Thus, EMD is not unique and the sifting algorithm can yield different numbers of IMF components for small changes to the stopping criteria. Noise assisted EMD or Ensemble EMD [3] addresses some of these issues but its computational complexity is a significant disadvantage.

A lack of rigorous mathematical treatment regarding the significance of extracted IMFs implies that many existing
EMD based fusion techniques are not based on any optimality criterion and often rely on visual inspection to determine 'relevant' IMFs. Only recently has research concentrated on automated procedures for EMD fusion. A partial reconstruction approach (PREMD) for the purposes of signal restoration, or denoising, has been proposed in [4]. It follows the behaviour of EMD as a dyadic filter bank for white Gaussian noise [5],[6], and the fact that the IMF log-variance follows a simple linear model controlled by the Hurst exponent. In [4], the use of confidence intervals which determine the level of noise energy in each of the IMF components was discussed for fusion by simply omitting IMFs dominated by noise energy. Although effective, the method is not based on any optimality criterion.

A recent solution for signal approximation using EMD followed by a weighting of IMFs was proposed in [7]. Two extensions of the algorithm were provided which fused the IMF components to produce the best estimate of the original signal in the least squares sense. The first, termed optimal EMD (OEMD), determined a set of weights for each of the IMF components so as to approximate the signal by linear combination. The other algorithm, termed bidirectional optimal EMD (BOEMD), takes into account the temporal structure of the signal and determines an optimal two-dimensional weight matrix so as to facilitate approximation by window based filtering. The advantages to this approach include enhanced performance over partial reconstruction in applications such as signal denoising, however, OEMD and BOEMD are limited by their block based nature. The relevance of the IMFs typically change with time and an adaptive approach would be more appropriate for real world signals.

Despite the inherent "fission" properties of EMD (that is the decomposition of a signal in hand into a number of oscillatory IMF components), the way to optimally fuse these features remains unclear. Most IMF fusion approaches are not based on optimality criteria, and typically relevant IMFs are selected by visual inspection or empirically (by applying binary weighting of IMFs). Other techniques, such as OEMD and BOEMD, perform optimisation in a least squares sense, but are not capable of local feature fusion due to their static nature. Adaptive algorithms are therefore not only desirable, but also necessary in order to make use of the full potential of EMD. In addition, fusion must be robust to some indetermi- 
nacies within EMD, such as sensitivity to parameter selection. Given that machine learning facilitates optimal and adaptive processing at the data level [8], it would therefore be beneficial to combine EMD and machine learning to perform fusion of the IMFs in an automated way that is based on a predefined optimality criterion. The simplest and best understood machine learning technique is adaptive filtering, which is used in this work in order to enhance the performance and scope of EMD.

\section{THE EMD ALGORITHM}

Empirical mode decomposition [1] is a technique to adaptively decompose a given signal, by means of a process called the sifting algorithm, into a finite set of AM/FM modulated components. These components, called "intrinsic mode functions" (IMFs), represent the oscillation modes embedded in the data. By definition, an IMF is a function for which the number of extrema and the number of zero crossings differ by at most one, and the mean of the two envelopes associated with the local maxima and local minima is approximately zero. The decomposition of a signal $x(k)$ is given by

$$
x(k)=\sum_{i=1}^{M} c_{i}(k)+r(k)
$$

where $c_{i}(k), i=1, \ldots, M$, is the set of IMFs and $r(k)$ is the residual. The first IMF is obtained as follows [1].

1. Let $\tilde{x}(k)=x(k)$;

2. Identify all local maxima and minima of $\tilde{x}(k)$;

3. Find an "envelope," $e_{\min }(k)$ (resp. $\left.e_{\max }(k)\right)$ that interpolates all local minima (resp. maxima);

4. Extract the "detail," $d(k)=x(k)-(1 / 2)\left(e_{\min }(k)+\right.$ $\left.e_{\max }(k)\right)$;

5. Let $\tilde{x}(k)=d(k)$ and go to step 2$)$; repeat until $d(k)$ becomes an IMF.

Once the first IMF is obtained, the procedure is applied to the residual $r(k)=x(k)-d(k)$ to obtain the second IMF. In this way, the procedure is applied recursively to obtain all the IMFs. An example of EMD is shown in Fig. 1 which illustrates a segment of speech (denoted by $U$ at the top of the figure) and its subsequent IMFs.

\section{PROPOSED ALGORITHM}

The architecture of the proposed machine learning enhanced EMD is shown in Fig. 2. Consider a 1D signal, $x(k)$, which by EMD is decomposed into $M$ IMF components $c_{i}(k)$. For each time instant $\mathrm{k}$, we construct a set of vectors, $\mathbf{c}(k)=$ $\left[c_{1}(k), \ldots, c_{M}(k)\right]^{T}$ where $(\cdot)^{T}$ denotes the matrix transpose operator. For convenience, the residue is included as $c_{M}(k)$.

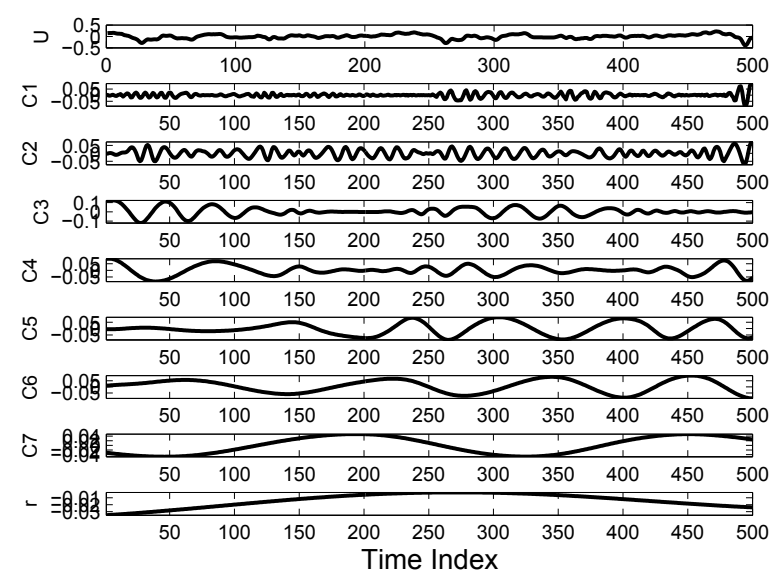

Fig. 1. Signal decomposition via EMD

The output, $y(k)$, of the proposed machine learning and EMD at time instant $k$ is given by

$$
y(k)=\operatorname{trace}\left[\mathbf{C}(k) \mathbf{W}^{T}(k)\right]
$$

where $\mathbf{C}(k)=[\mathbf{c}(k), \ldots, \mathbf{c}(k-N+1)]$ is the "fission" matrix comprised of a spatio-temporal window over the IMFs, W $(k)$ is a $M \times N$ weight matrix, $N$ is the filter length of the adaptive filter and the trace of a matrix is the sum of all the elements on the main diagonal. We propose to update $\mathbf{W}(\mathrm{k})$ as

$$
\begin{aligned}
e(k) & =\hat{d}(k)-y(k) \\
\mathbf{W}(k+1) & =\mathbf{W}(k)+\eta(k) e(k) \mathbf{C}(k) \\
\eta(k) & =\frac{\mu}{\operatorname{trace}\left[\mathbf{C}(k) \mathbf{C}^{T}(k)\right]+\epsilon}
\end{aligned}
$$

where $e(k)$ is the instantaneous error at the output of the filter, $\hat{d}(k)$ is the desired or training signal, and $\mu$ is the learning rate, $0<\mu<2$. The regularisation parameter $\epsilon$ is included so as to prevent the algorithm from becoming unstable when trace $\left[\mathbf{C}(k) \mathbf{C}^{T}(k)\right]$ is close to zero. Note our proposed algorithm uses a normalised least mean square (NLMS) like algorithm to update the weight matrix, $\mathbf{W}$, but it can easily be extended to incorporate any adaptive filtering algorithm. We shall hereby refer to the proposed algorithm as NLMSEMD. From eqn. (3), NLMS-EMD takes values from every

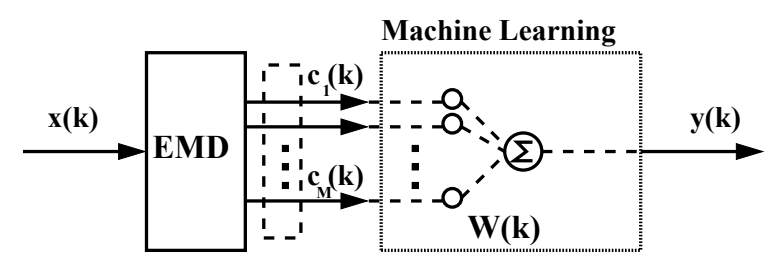

Fig. 2. Machine learning enhanced EMD

IMF within a given spatial dimension defined by parameter 
$N$. If $N=1$, the algorithm is equivalent to the static case of OEMD except that our algorithm is adaptive and hence much more flexible. Depending on the dynamics and correlation structure of the input, $N$ may be increased to capture its temporal nature. Similarly if the input satisfies the criteria of an IMF, the number of IMFs would be equal to unity $(M=1)$, and the algorithm would be equivalent to the NLMS with a filter length of $N$.

The proposed extension of EMD is fully adaptive to facilitate local feature relevance and automatic fusion, which is of particular importance for nonlinear and nonstationary data. The robust nature of the algorithm means it is less sensitive to changes in EMD parameters and, in addition, it is fast and has low computational complexity.

\section{SIMULATIONS}

We illustrate the potential of the proposed NLMS-EMD algorithm on case studies of signal denoising and prediction. To provide insight into the performance of NLMS-EMD, we performed simulations using a selection of real world signals such as speech and biomedical data, as well as benchmark nonlinear and linear signals. Performance comparisons were made with standard NLMS filtering, PREMD and OEMD.

\subsection{Denoising}

In the first experiment the original signal was a segment of electroencephalogram (EEG) data. White Gaussian noise was added so that the signal to noise ratio (SNR) was $13.4 \mathrm{~dB}$, where SNR is defined by

$$
\mathrm{SNR}=10 \log _{10} \sum_{k} \frac{\hat{d}^{2}(k)}{(\hat{d}(k)-y(k))^{2}} \mathrm{~dB}
$$

Table 1 illustrates the quantative performance of the algorithms and segments of the reconstructed signals are shown in Fig. 3 [(a),(b),(c) and (d)]. The filter length of NLMSEMD was chosen as $M \times 1$ so as to make a fair comparison with OEMD. Additionally, only an optimal filter length within the range $1-200$ was considered for NLMS, since typically $M<<200$. The number of IMFs obtained from the decomposition of the EEG signal was 13 and accordingly the filter length of NLMS-EMD was $13 \times 1$. The best learning rate for the proposed algorithm was found to be $\mu=1.33$. The best learning for NLMS was determined to be $\mu=1.52$ with $N=180$. From Table 1, the quantative performance of NLMS-EMD exceeds OEMD and NLMS by at least $4 \mathrm{~dB}$, which is visualised in Fig. 3(a) - Fig. 3(d). It is clear that its adaptive and robust nature allow it to track fast changes in signal dynamics to a high degree of accuracy. Fig. 3(e) and Fig. 3(f) show respectively the trajectory of some of the weight coefficients for OEMD and NLMS-EMD, observe the static "optimal" weights for OEMD, and the dynamic weight

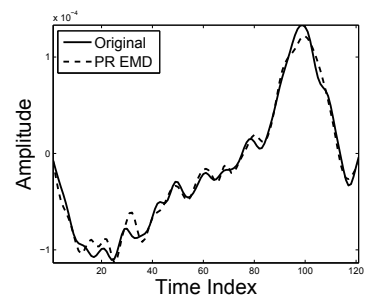

(a)

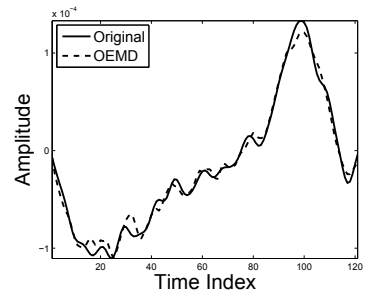

(c)

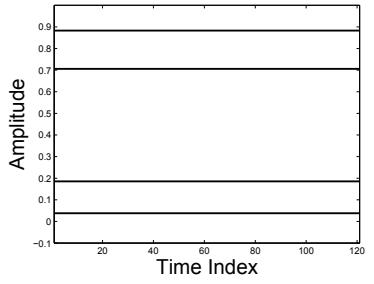

(e)

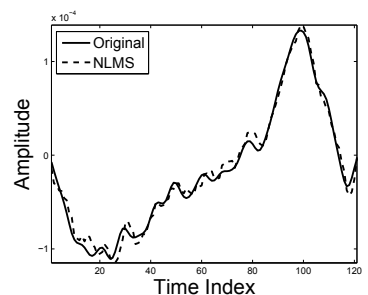

(b)

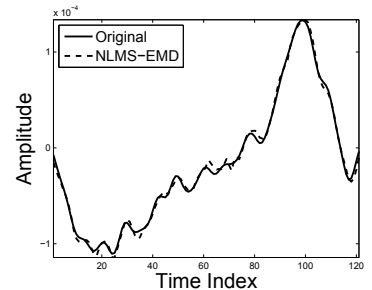

(d)

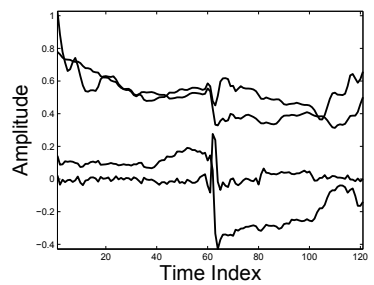

(f)
Fig. 3. Denoising results. (a) PREMD, (b) NLMS, (c) OEMD, (d) NLMS-EMD. The weight coefficients for the first four IMF components of OEMD and NLMS-EMD are shown in (e) and (f) respectively, note the dynamic nature of NLMSEMD compared to the static OEMD.

Table 1. SNR denoising performances of the PREMD, OEMD, NLMS and EMD-NLMS algorithms for Fig. 3.

\begin{tabular}{|c|c|c|c|}
\hline PREMD & OEMD & NLMS & NLMS-EMD \\
\hline $17.8 \mathrm{~dB}$ & $19.2 \mathrm{~dB}$ & $19 \mathrm{~dB}$ & $24 \mathrm{~dB}$ \\
\hline
\end{tabular}

behaviour within NLMS-EMD, which emphasizes the adaptive nature of the proposed algorithm over OEMD. Due to its block based nature, OEMD cannot track changing signal dynamics, which makes it suboptimal for nonlinear and nonstationary inputs. In the second experiment, the denoising capabilities of NLMS-EMD were further demonstrated for image restoration. The original image was subjected to additive white Gaussian noise so as to introduce an SNR of $18.5 \mathrm{~dB}$. The resulting SNR of the restored image was $24 \mathrm{~dB}$ compared to $22.3 \mathrm{~dB}$ for the OEMD algorithm. In addition to an improvement in quantative performance, there was an improvement in the perceptual quality of the restored image using NLMS-EMD. 
Table 2. Prediction performances (in SNR) of the NLMS and EMD-NLMS algorithms.

\begin{tabular}{|c|c|c|}
\hline Signal & NLMS & NLMS-EMD \\
\hline AR(4) & $8 \mathrm{~dB}$ & $11.5 \mathrm{~dB}$ \\
Mackey Glass & $18.4 \mathrm{~dB}$ & $20.7 \mathrm{~dB}$ \\
Speech & $19.2 \mathrm{~dB}$ & $19.3 \mathrm{~dB}$ \\
\hline
\end{tabular}

\subsection{Prediction}

We next demonstrate the application of EMD combined with machine learning to signal prediction, particularly in processing nonlinear signals or signals with large dynamics. We performed prediction on several signals: a coloured noise series generated from a stable AR(4)-model (used as our benchmark linear signal) denoted by $x_{\mathbf{A R}}$ where $v(k)$ is a white noise source with standard normal distribution;

$$
\begin{aligned}
& x_{\mathbf{A R}}(k)=\quad \begin{array}{c}
1.79 x_{\mathbf{A R}}(k-1)-1.85 x_{\mathbf{A R}}(k-2) \\
+1.27 x_{\mathbf{A R}}(k-3)-0.41 x_{\mathbf{A R}}(k-4)+v(k)
\end{array}
\end{aligned}
$$

a realization of the Mackey Glass equation given by (5) (used as our benchmark non-linear signal);

$$
\frac{d x}{d k}=\frac{0.2 x(k-\tau)}{1+x^{10}(k-\tau)}-0.1 x(k)
$$

and a real world signal, a segment of speech sampled at 48000 Hz. The quantative prediction performance is shown in Table 2. The results indicate that for both the benchmark (linear and nonlinear) signals and speech, the NLMS-EMD algorithm outperformed standard NLMS. This is further illustrated by Fig. 4 which shows a segment of the prediction results for the Mackey Glass series. Note that the NLMS-EMD based prediction produced results that are better aligned with the original signal than the NLMS.

\section{CONCLUSIONS}

We have introduced an extension of the EMD algorithm that uses machine learning techniques to enhance its performance. Unlike previous extensions, the approach is fully adaptive to cater for the nonstationary and nonlinear nature of real world data. Based on the "data fusion via fission" property of EMD, the machine learning facilitates a real-time assessment of the component (IMF) relevance. The proposed extension makes the EMD robust to changes in stopping criteria as well as errors in the envelope estimation process, making it suitable for data fusion. The simulation results demonstrate the robust and adaptive nature of the algorithm.

\section{REFERENCES}

[1] N. E. Huang, Z. Shen, S. R. Long, M. L. Wu, H. H. Shih, Z. Quanan, N. C. Yen, C. C. Tung, and H. H. Liu, "The empirical mode decomposition and the Hilbert spectrum

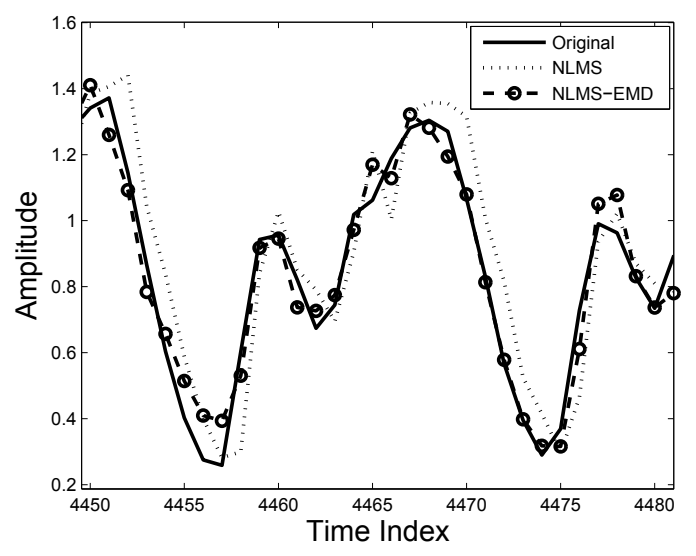

Fig. 4. Adaptive prediction results of the Mackey Glass time series. The NLMS-EMD based results are better aligned with the original signal than the NLMS.

for nonlinear and non-stationary time series analysis," Proceedings of the Royal Society of London Series A, vol. 454, pp. 903-995, 1998.

[2] D. P. Mandic, M. Golz, A. Kuh, D. Obradovic, and T. Tanaka, Signal Processing Techniques for Knowledge Extraction and Information Fusion, Springer, 2007.

[3] Z. Wu and N. E. Huang, "Ensemble empirical mode decomposition: a noise-assisted data analysis method," Tech. Rep. 193, Center for Ocean-Land-Atmosphere Studies, 2004.

[4] P. Flandrin, P. Goncalves, and G. Rilling, "Detrending and denoising with empirical mode decompositions," in Proceedings of the European signal processing conference (EUSIPCO'04), September 2004, vol. 2, pp. 15811584.

[5] Z. Wu and N. E. Huang, "A study of the characteristics of white noise using the empirical mode decomposition method," Proceedings of the Royal Society of London Series A, vol. 460, pp. 1597-1611, june 2004.

[6] P. Flandrin, G. Rilling, and P. Goncalves, "Empirical mode decomposition as a filter bank," IEEE Signal Processing Letters, vol. 11, no. 2, pp. 112-114, February 2004.

[7] B. Weng and K. E. Barner, "Optimal and bidirectional optimal empirical mode decomposition," in Proceedings of the IEEE International Conference on Acoustics, Speech, and Signal Processing (ICASSP'07), 2007, vol. III, pp. 1501-1504.

[8] A. Cichocki and S. I. Amari, Blind Signal and Image Processing, Wiley, 2002. 